Supplement of Atmos. Chem. Phys., 19, 7667-7690, 2019

https://doi.org/10.5194/acp-19-7667-2019-supplement

(c) Author(s) 2019. This work is distributed under

the Creative Commons Attribution 4.0 License.

(c) (1)

Supplement of

\title{
Emission of volatile halogenated organic compounds over various Dead Sea landscapes
}

Moshe Shechner et al.

Correspondence to: Eran Tas (eran.tas@mail.huji.ac.il)

The copyright of individual parts of the supplement might differ from the CC BY 4.0 License. 
33 S1. Lifetime and main sink pathways for the investigated VHOCs

34 Table S1. Average lifetimes and primary removal pathways for the VHOCs ${ }^{\mathrm{a}}$.

35

\begin{tabular}{|c|c|c|}
\hline Species & Average lifetime & Primary removal pathway \\
\hline $\mathrm{CHBr}_{3}$ & 15 days & $\mathrm{J}^{\mathrm{c}}>\mathrm{OH} \quad 36$ \\
\hline $\mathrm{CH}_{2} \mathrm{Br}_{2}$ & 94 days & $\mathrm{OH}$ \\
\hline $\mathrm{CHBr}_{2} \mathrm{Cl}$ & 32 days & $\mathrm{OH}>\mathrm{J}$ \\
\hline $\mathrm{CHBrCl}_{2}$ & 41 days & $\mathrm{OH}>\mathrm{J}$ \\
\hline $\mathrm{C}_{2} \mathrm{HCl}_{3}$ & 5 days & $\mathrm{OH}$ \\
\hline $\mathrm{CHCl}_{3}$ & 112 days & $\mathrm{OH}$ \\
\hline $\mathrm{CH}_{3} \mathrm{I}$ & 4 days & $\mathrm{J}>\mathrm{OH}$ \\
\hline $\mathrm{CH}_{3} \mathrm{Br}$ & 1.8 years $^{b}$ & $\mathrm{OH}>$ loss in soil>loss in ocean \\
\hline $\mathrm{CH}_{3} \mathrm{Cl}$ & 1.57 years $^{b}$ & $\mathrm{OH}>$ loss in ocean>loss in soil \\
\hline
\end{tabular}

45

$46 \quad{ }^{a}$ See more details in (Carpenter et al., 2014).

$47 \quad{ }^{\mathrm{b}}$ Partial lifetime against reaction with $\mathrm{OH}$.

$48 \quad{ }^{\mathrm{c}} \mathrm{J}$, photolysis first-order rate coefficient. 
Table S2. Specification of instrumentation and techniques used to analyze the sampled gas and corresponding precision.

53

54

\begin{tabular}{|c|c|c|c|}
\hline Species & System $^{a}$ & \multicolumn{2}{|c|}{ Precision $(\% 5)^{5}$} \\
\hline \multirow[t]{2}{*}{$\mathrm{CHBr}_{3}$} & \multirow[t]{2}{*}{$\mathrm{D}, \mathrm{MSD}$} & \multirow[t]{2}{*}{1.6} & 56 \\
\hline & & & 57 \\
\hline $\mathrm{CH}_{2} \mathrm{Br}_{2}$ & D & 1.6 & 58 \\
\hline \multirow{2}{*}{$\mathrm{CHBr}_{2} \mathrm{Cl}$} & $\mathrm{D}$ & 9.3 & 59 \\
\hline & \multirow[b]{2}{*}{$\mathrm{D}$} & \multirow[b]{2}{*}{2.0} & 61 \\
\hline $\mathrm{CHBrCl}_{2}$ & & & 62 \\
\hline \multirow[t]{2}{*}{$\mathrm{CH}_{2} \mathrm{Cl}_{2}$} & \multirow[t]{2}{*}{ C, MSD } & \multirow[t]{2}{*}{4.5} & 63 \\
\hline & & & 64 \\
\hline $\mathrm{C}_{2} \mathrm{HCl}_{3}$ & \multirow[t]{2}{*}{ MSD } & \multirow[t]{2}{*}{$\leq 5.0$} & 65 \\
\hline \multirow{2}{*}{$\mathrm{CHCl}_{3}$} & & & 66 \\
\hline & $\mathrm{C}, \mathrm{D}, \mathrm{MSD}$ & 1.1 & $6 /$ \\
\hline \multirow[t]{2}{*}{$\mathbf{C H}_{3} \mathbf{I}$} & \multirow[t]{2}{*}{ C,D } & \multirow[t]{2}{*}{1.1} & 68 \\
\hline & & & 69 \\
\hline \multirow[t]{2}{*}{$\mathrm{CH}_{3} \mathrm{Br}$} & \multirow[t]{2}{*}{ MSD } & \multirow[t]{2}{*}{1.7} & 70 \\
\hline & & & 71 \\
\hline $\mathrm{CH}_{3} \mathrm{Cl}$ & B, MSD & 1.5 & $\begin{array}{l}72 \\
73\end{array}$ \\
\hline
\end{tabular}

${ }^{\mathrm{a}}$ Indicates the applied column and detector for quantifying species concentration: B, DB-1 column + flame

75 ionization detector (FID); C, DB-5 column + electron-capture detector (ECD); D, RESTEK1701 column + ECD;

76 MSD, DB-5 MS column + mass spectrometer. 


\section{S3. Measured VHOC mixing ratios}

78 Table S3 compares the measured mixing ratios at the different measurement sites and reported

79 values for the global marine boundary layer (MBL).

80

81 Table S3. Comparison of VHOC mixing ratios (in pptv) measured at the Dead Sea to their corresponding values

82 at the marine boundary layer (MBL). Unless otherwise specified, the table presents median, minimum and

83 maximum VHOC mixing ratios measured at different sites at the Dead Sea (see Table 1 in the main text for site

84 abbreviations) and in the MBL as reported by Carpenter et al. (2014).

\begin{tabular}{|c|c|c|c|c|c|c|c|c|c|c|}
\hline Species & $\begin{array}{l}\text { Median/ } \\
\text { range }\end{array}$ & $\begin{array}{l}\text { BARE- } \\
\text { MSMR }\end{array}$ & $\begin{array}{c}\text { BARE- } \\
\text { MSD }\end{array}$ & $\begin{array}{c}\text { COAST- } \\
\text { EGD }\end{array}$ & $\begin{array}{c}\text { COAST- } \\
\text { TKM }\end{array}$ & $\begin{array}{c}\text { TMRX- } \\
\text { ET }\end{array}$ & $\begin{array}{l}\text { WM- } \\
\text { KLY }\end{array}$ & $\begin{array}{l}\text { SEA- } \\
\text { KDM }\end{array}$ & All sites & MBL \\
\hline \multirow[b]{2}{*}{$\mathrm{CHBr}_{3}$} & Median & 11.3 & 11.0 & 8.0 & 2.6 & 4.7 & 3.1 & 11.0 & 6.2 & 1.2 \\
\hline & Range & $5.6-16.3$ & $6.0-22.6$ & $4.4-16.8$ & $3.6-1.9$ & $2.9-7.1$ & $2.3-3.5$ & $5.4-16.5$ & $1.9-22.6$ & 0.4-4.0 \\
\hline \multirow{2}{*}{$\mathrm{CH}_{2} \mathrm{Br}_{2}$} & Median & 0.9 & 2.7 & 1.8 & 0.8 & 1.3 & 1.1 & 1.4 & 1.1 & 0.9 \\
\hline & Range & $0.9-1.0$ & $0.7-18.6$ & $0.9-5.1$ & $0.9-0.8$ & $0.9-1.6$ & $1.0-1.2$ & $1.1-1.7$ & $0.7-18.6$ & 0.6-1.7 \\
\hline \multirow{2}{*}{$\mathrm{CHBr}_{2} \mathrm{Cl}$} & Median & 4.8 & 4.1 & 2.2 & 1.2 & 2.2 & 0.9 & 1.3 & 2.4 & 0.3 \\
\hline & Range & $3.2-5.4$ & $2.2-11.0$ & $0.4-6.5$ & $7.5-0.5$ & $0.6-3.6$ & $0.5-1.0$ & $0.5-2.2$ & 0.4-11.0 & $\begin{array}{l}0.1-0.8 \\
\end{array}$ \\
\hline \multirow{2}{*}{$\mathrm{CHBrCl}_{2}$} & Median & 2.6 & 2.5 & 1.6 & 1.0 & 2.4 & 0.9 & 2.0 & 1.4 & 0.3 \\
\hline & Range & $2.6-3.7$ & $0.9-9.6$ & $1.0-3.0$ & $0.5-1.4$ & $0.7-3.9$ & $0.6-1.1$ & $1.3-2.7$ & $0.5-9.6$ & 0.1-0.9 \\
\hline \multirow{2}{*}{$\mathrm{C}_{2} \mathrm{HCl}_{3}$} & Median & 1.15 & 1.7 & 1.3 & 1.6 & 1.1 & 2.7 & 1.2 & 1.5 & 0.5 \\
\hline & Range & $0.84-1.22$ & $1.0-2.7$ & $0.3-10.5$ & $0.4-2.9$ & $0.4-1.5$ & $1.0-4.1$ & $0.8-1.6$ & $0.4-10.5$ & $0.05-2$ \\
\hline \multirow{2}{*}{$\mathrm{CHCl}_{3}$} & Median & 16.9 & 19.8 & 18.2 & 18.7 & 19.0 & 19.8 & 17.3 & 18.63 & 7.5 \\
\hline & Range & $15.9-20.5$ & $18.8-25.3$ & $14.5-27.9$ & $15.4-20.1$ & $18.4-57.2$ & $18.8-5.3$ & $16.5-18.2$ & $14.5-57.2$ & 7.3-7.8 \\
\hline \multirow{2}{*}{$\mathrm{CH}_{3} \mathrm{I}$} & Median & 0.8 & 1.3 & 1.2 & 1.5 & 1.3 & 1.1 & 1.4 & 1.2 & 0.8 \\
\hline & Range & $0.8-0.8$ & $1.0-1.5$ & $0.4-2.1$ & $1.5-1.2$ & $0.8-2.8$ & $0.7-1.6$ & $1.2-1.6$ & $0.4-2.8$ & 0.3-2.1 \\
\hline \multirow{2}{*}{$\mathrm{CH}_{3} \mathrm{Br}$} & Mean & 8.6 & 10.6 & 9.29 & 9.12 & 10.2 & 8.45 & 9.65 & 9.1 & $7.01^{\mathrm{a}}$ \\
\hline & Range & $8.1-9.4$ & $7.8-13.8$ & $7.5-13.3$ & $8.5-7.5$ & $8.3-13.8$ & $7.8-9.2$ & $9.1-10.2$ & 7.5-13.8 & $6.95-7.07^{\mathrm{a}}$ \\
\hline \multirow{2}{*}{$\mathrm{CH}_{3} \mathrm{Cl}$} & Mean & 601 & 592 & 617 & 614 & 744 & 633 & 596 & 601 & $540^{\mathrm{a}}$ \\
\hline & Range & $583-608$ & $549-672$ & $531-732$ & $583-545$ & $581-685^{*}$ & $591-668$ & $583-608$ & 531-732* & $537-542^{\mathrm{a}}$ \\
\hline
\end{tabular}

85

$86{ }^{a}$ Mean and range for 2012 based on flask measurements by the US National Oceanic and Atmospheric

87 Administration (NOAA) (http://www.esrl.noaa.gov/gmd/dv/site/) and in situ measurements by the Advanced

88 Global Atmospheric Gases Experiment (AGAGE) (http://agage.eas.gatech.edu/). Measurements are taken from

89 ground stations, which do not represent the MBL in all cases. See Table 1 in the main text for site abbreviations.

90 *Calculation excludes one $\mathrm{CH}_{3} \mathrm{Cl}$ measurement at TMRX-ET-1 (see Sect. 2.1.2 in main text). 
91 Figure S1 compares the mixing ratios of the measured VHOCs at different distances from the

92 seawater, and individually for winter and spring. No clear impact of season or distance from the

93 seawater on the mixing ratios can be discerned in this figure, or for the sample taken at

94 SEA-KDM, the latter directly representing air masses over the seawater (Sect. 2.1.1 in the main

text). Additional discussion of these results is included in Sect. 3.2.1 in the main text.
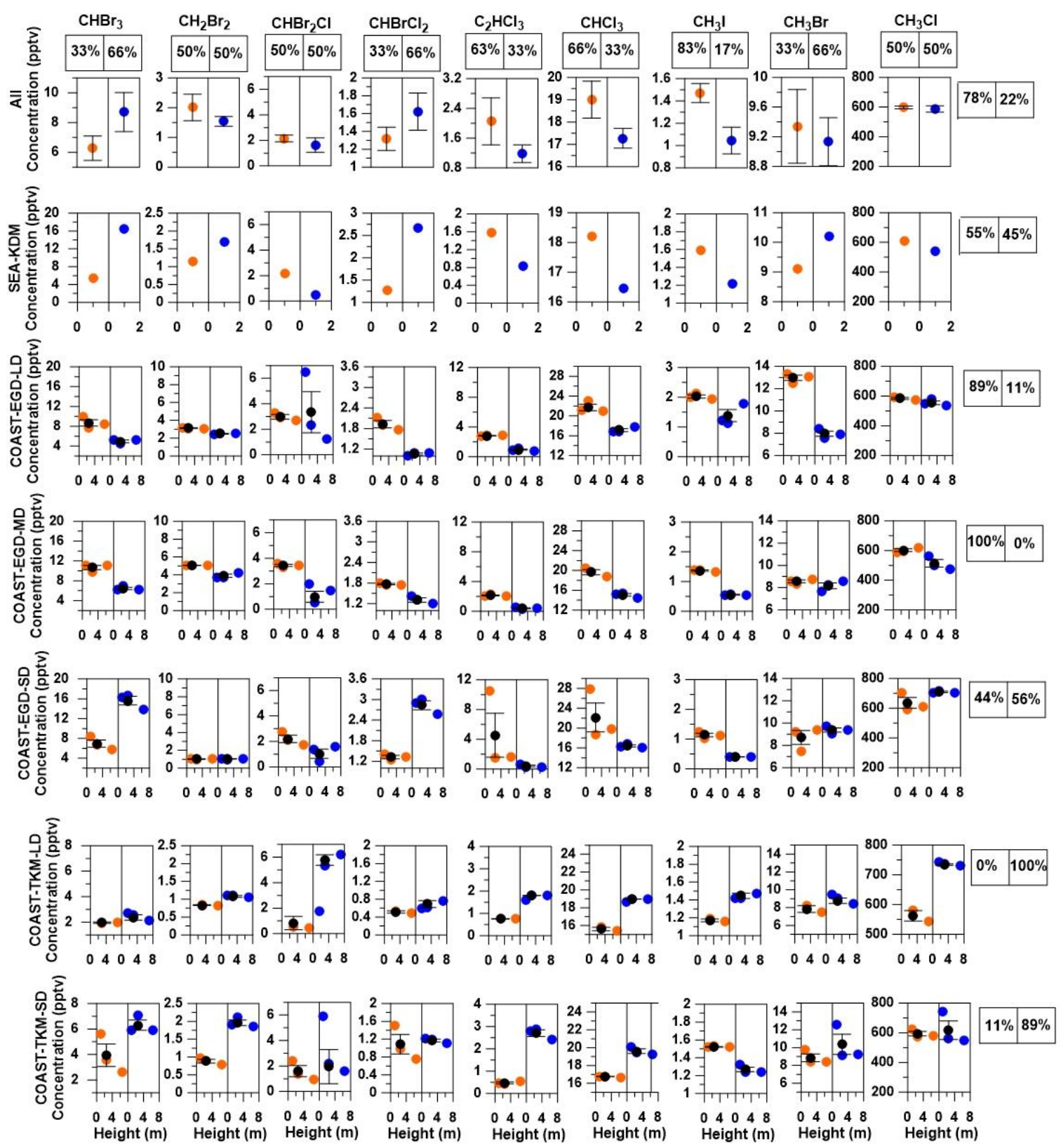

Spring

Winter

97 Figure S1. Seasonal and spatial influences on measured mixing ratios of VHOCs. Measured VHOC mixing ratios are presented vs. vertical height above surface level, separately for winter (blue) and spring (orange). Black filled circles and error bars represent average and standard error of the mean, respectively. Values above and to 
the right of the figure indicate the percentage of time during which average mixing ratios were higher in the spring

101 (left box) or in the winter (right box), individually for COAST-TKM, COAST-EGD and SEA-KDM sites, and

102 for all of these sites together (All), for all sites and all species, respectively (see Table 1 in the main text for

103 measurement site abbreviations).

104

\section{S4. Sampling footprints}

106

Table S4. Calculated footprint following the methodology of Schuepp et al. (1990). The table shows the date, (high) canister during each measurement.

\begin{tabular}{|c|c|c|c|c|c|c|c|}
\hline $\begin{array}{c}\text { Date } \\
\text { dd/m/yyyy }\end{array}$ & $\begin{array}{l}\text { Time } \\
\text { (local) }\end{array}$ & $\begin{array}{c}\text { Site name / } \\
\text { measurement } \\
\text { abbreviation }^{\text {a }}\end{array}$ & $\begin{array}{l}\text { Sampling } \\
\text { height } \\
\text { (m) }\end{array}$ & $\begin{array}{c}80 \% \\
\text { footprint } \\
\text { low (m) }\end{array}$ & $\begin{array}{c}\mathbf{8 0 \%} \\
\text { footprint } \\
\text { high }(\mathrm{m})\end{array}$ & $\begin{array}{c}90 \% \\
\text { footprint } \\
\text { low (m) }\end{array}$ & $\begin{array}{c}90 \% \\
\text { footprint } \\
\text { high (m) }\end{array}$ \\
\hline $20 / 4 / 2016$ & $\begin{array}{c}08: 45- \\
08: 55\end{array}$ & $\begin{array}{l}\text { BARE-MSMR / } \\
\text { BARE-MSMR-1 }\end{array}$ & $2.5,4.5,7.0$ & 293.5 & 954.9 & 621.7 & 2022.3 \\
\hline $21 / 4 / 2016$ & $\begin{array}{c}08: 45- \\
08: 55\end{array}$ & $\begin{array}{l}\text { WM-KLY / } \\
\text { WM-KLY-1 }\end{array}$ & $1.0,2.0,4.0$ & 105.7 & 502.6 & 223.9 & 1064.5 \\
\hline $02 / 5 / 2016$ & $\begin{array}{c}08: 45- \\
08: 55\end{array}$ & $\begin{array}{l}\text { TMRX-ET / } \\
\text { TMRX-ET-1 }\end{array}$ & $4.5,5.5,7.5$ & 135.6 & 444.2 & 287.2 & 940.8 \\
\hline $03 / 5 / 2016$ & $\begin{array}{l}08: 45- \\
08: 55\end{array}$ & $\begin{array}{l}\text { WM-KLY / } \\
\text { WM-KLY-2 }\end{array}$ & $1.0,2.0,4.0$ & 105.7 & 502.6 & 223.9 & 1064.5 \\
\hline $25 / 5 / 2016$ & $\begin{array}{c}08: 30- \\
08: 40\end{array}$ & $\begin{array}{l}\text { BARE-MSD / } \\
\text { BARE-MSD-1 }\end{array}$ & $1.25,2.5,5$ & 126.2 & 643.3 & 267.3 & 1362.4 \\
\hline $26 / 5 / 2016$ & $\begin{array}{c}08: 30- \\
08: 40\end{array}$ & $\begin{array}{l}\text { BARE-MSD / } \\
\text { BARE-MSD-2 }\end{array}$ & $1.25,2.5,5$ & 126.2 & 643.3 & 267.3 & 1362.4 \\
\hline $30 / 5 / 2016$ & $\begin{array}{c}12: 00- \\
12: 10\end{array}$ & $\begin{array}{l}\text { TMRX-ET / } \\
\text { TMRX-ET-2 }\end{array}$ & $4.5,5.5,7.5$ & 135.6 & 444.2 & 287.2 & 940.8 \\
\hline $31 / 5 / 2016$ & $\begin{array}{l}12: 00- \\
12: 10\end{array}$ & $\begin{array}{l}\text { BARE-MSMR / } \\
\text { BARE-MSMR-2 }\end{array}$ & $2.5,4.5,7$ & 293.5 & 954.9 & 621.7 & 2022.3 \\
\hline $11 / 7 / 2016$ & $\begin{array}{l}12: 00- \\
12: 20\end{array}$ & $\begin{array}{l}\text { BARE-MSD / } \\
\text { BARE-MSD-3 }\end{array}$ & $1.25,2.5,5$ & 126.2 & 643.3 & 267.3 & 1362.4 \\
\hline $11 / 7 / 2016$ & $\begin{array}{c}18: 00^{-} \\
18: 20\end{array}$ & $\begin{array}{l}\text { BARE-MSD / } \\
\text { BARE-MSD-4 }\end{array}$ & $1.25,2.5,5$ & 126.2 & 643.3 & 267.3 & 1362.4 \\
\hline $21 / 2 / 2017$ & $\begin{array}{c}11: 20- \\
11: 40\end{array}$ & $\begin{array}{l}\text { COAST-TKM-SD / } \\
\text { COAST-TKM-SD-w }\end{array}$ & $1,2.5,6.5$ & 126.8 & 932.0 & 268.6 & 1973.9 \\
\hline $22 / 2 / 2017$ & $\begin{array}{l}11: 00^{-} \\
11: 20\end{array}$ & $\begin{array}{l}\text { COAST-TKM-LD / } \\
\text { COAST-TKM-LD-w }\end{array}$ & $1.5,3,7$ & 94.1 & 717.5 & 199.3 & 1519.6 \\
\hline $28 / 2 / 2017$ & $\begin{array}{l}11: 20- \\
11: 40\end{array}$ & $\begin{array}{l}\text { COAST-EGD-SD / } \\
\text { COAST-EGD-SD-w }\end{array}$ & $1,2.5,6.5$ & 126.8 & 932.0 & 268.6 & 1973.9 \\
\hline $01 / 3 / 2017$ & $\begin{array}{l}11: 07- \\
11: 27 \\
\end{array}$ & $\begin{array}{l}\text { COAST-EGD-MD / } \\
\text { COAST-EGD-MD-w }\end{array}$ & $1,2.5,6.5$ & 126.8 & 932.0 & 268.6 & 1973.9 \\
\hline $02 / 3 / 2017$ & $\begin{array}{c}11: 00- \\
11: 20\end{array}$ & $\begin{array}{l}\text { COAST-EGD-LD / } \\
\text { COAST-EGD-LD-w }\end{array}$ & $1,2.5,6.5$ & 126.8 & 932.0 & 268.6 & 1973.9 \\
\hline $25 / 4 / 2017$ & $\begin{array}{l}11: 30- \\
11: 50\end{array}$ & $\begin{array}{c}\text { COAS-EGD-SD / } \\
\text { COAST-EGD-SD-s }\end{array}$ & $1,2.5,6.5$ & 126.8 & 932.0 & 268.6 & 1973.9 \\
\hline $26 / 4 / 2017$ & $\begin{array}{l}11: 00- \\
11: 20\end{array}$ & $\begin{array}{l}\text { COAST-EGD-MD / } \\
\text { COAST- EGD-MD-s }\end{array}$ & $1,2.5,6.5$ & 98.3 & 722.5 & 208.2 & 1530.2 \\
\hline $27 / 4 / 2017$ & $\begin{array}{l}11: 00- \\
11: 20\end{array}$ & $\begin{array}{l}\text { COAST-EGD-LD / } \\
\text { COAST-EGD-LD-s }\end{array}$ & $1,2.5,6.5$ & 126.8 & 932.0 & 268.6 & 1973.9 \\
\hline $03 / 5 / 2017$ & $\begin{array}{l}12: 10- \\
12: 30\end{array}$ & $\begin{array}{l}\text { COAST-TKM-SD / } \\
\text { COAST-TKM-SD-s }\end{array}$ & $1,2.5,6.5$ & 126.8 & 932.0 & 268.6 & 1973.9 \\
\hline $04 / 5 / 2017$ & $\begin{array}{c}10: 30- \\
10: 50\end{array}$ & $\begin{array}{l}\text { COAST-TKM-LD / } \\
\text { COAST-TKM-LD-s }\end{array}$ & $1.5,3,7$ & 94.1 & 717.5 & 199.3 & 1519.6 \\
\hline
\end{tabular}


a Suffixes "s" and "w" refer to samples taken during the spring and winter, respectively. SD, MD, and LD refer to relatively short, medium, and long distance from the coastline, respectively (see Sect. 2.1 in the main text).

112 Additional abbreviations: MSD, Masada; MSMR, Mishmar; KLY, Kalya; ET, Ein Tamar; KDM, Kedem; EGD,

113 Ein Gedi; BARE, bare soil site; COAST, coastal soil-salt mixture site; WM, agricultural watermelon-cultivation 114 site; TMRX, natural Tamarix site; SEA, sampling near the seawater (see Sect. 2.1.1 in the main text).

\section{S5. Anthropogenic influence during the measurements}

117 We used the measured mixing ratios of $\mathrm{C}_{2} \mathrm{HCl}_{3}$ and $\mathrm{C}_{2} \mathrm{Cl}_{4}$ to explore potential influences on the sampled air composition, considering that these two species typically have a dominant anthropogenic origin (Zhou et al., 2005). Table S5 presents the Pearson correlation coefficients for the correlations between the VHOCs that were investigated in this work. The table indicates

121 a much higher correlation between $\mathrm{C}_{2} \mathrm{HCl}_{3}$ and $\mathrm{C}_{2} \mathrm{Cl}_{4}$ compared to all other correlations, 122 indicating an anthropogenic source for $\mathrm{C}_{2} \mathrm{HCl}_{3}(p<0.05)$.

Table S5. Pearson correlation coefficient for the correlation between the investigated VHOCs.

\begin{tabular}{|c|c|c|}
\hline & $\mathrm{C}_{2} \mathrm{HCl}_{3}$ & $\mathrm{C}_{2} \mathrm{Cl}_{4}$ \\
\hline $\mathrm{CHCl}_{3}$ & 0.27 & 0.27 \\
\hline $\mathrm{C}_{2} \mathrm{HCl}_{3}$ & 1.00 & 0.73 \\
\hline $\mathrm{C}_{2} \mathrm{Cl}_{4}$ & 0.73 & 1.00 \\
\hline $\mathrm{CH}_{3} \mathrm{Cl}$ & 0.04 & 0.12 \\
\hline $\mathrm{CH}_{3} \mathrm{Br}$ & 0.12 & 0.16 \\
\hline $\mathrm{CH}_{3} \mathrm{I}$ & 0.21 & 0.22 \\
\hline $\mathrm{CH}_{2} \mathrm{Br}_{2}$ & 0.00 & 0.01 \\
\hline $\mathrm{CHBrCl}_{2}$ & 0.04 & -0.12 \\
\hline $\mathrm{CHBr}_{2} \mathrm{Cl}$ & 0.15 & 0.12 \\
\hline $\mathrm{CHBr}_{3}$ & 0.02 & -0.11 \\
\hline
\end{tabular}

To further investigate the origin of $\mathrm{C}_{2} \mathrm{HCl}_{3}$ and $\mathrm{C}_{2} \mathrm{Cl}_{4}$, we plotted their measured mixing

127 ratios as well as the $\left[\mathrm{C}_{2} \mathrm{Cl}_{4}\right] /\left[\mathrm{C}_{2} \mathrm{HCl}_{3}\right]$ ratio vs. corresponding wind direction (Fig. S2). The ratio between the two species can be used to evaluate the transportation duration of the sampled air masses between the anthropogenic origin and the sampling point, considering the significantly longer atmospheric lifetime of $\mathrm{C}_{2} \mathrm{Cl}_{4}$ (3.5-4 months; (Olaguer, 2002;Singh et al., 
131 1996)), compared to $\mathrm{C}_{2} \mathrm{HCl}_{3}$ ( 7 days; (Quack and Suess, 1999)). It should be emphasized, 132 however, that the ratio $\left[\mathrm{C}_{2} \mathrm{Cl}_{4}\right] /\left[\mathrm{C}_{2} \mathrm{HCl}_{3}\right]$ may vary with time for a specific emission source, 133 and our measurements are also potentially exposed to more than a single anthropogenic origin.

134 The apparently dominant origin in the study area is, however, the Dead Sea Works (DSW), 135 located a few kilometers to the south of most measurement sites (see Fig. 1 in the main text). 136 Other potential emission sources were generally located at least a few dozen kilometers from 137 the study area. It should be further emphasized that Fig. S2a presents $\mathrm{C}_{2} \mathrm{Cl}_{4}$ and $\mathrm{C}_{2} \mathrm{HCl}_{3}$ mixing 138 ratios and $\left[\mathrm{C}_{2} \mathrm{Cl}_{4}\right] /\left[\mathrm{C}_{2} \mathrm{HCl}_{3}\right]$ collectively for all measurement sites located to the north of the 139 DSW. The justification for this is the relatively short distances between these sites, compared 140 to that between any of these sites and a potential origin for anthropogenic VHOC emission 141 other than the DSW. Fig. S2b presents $\mathrm{C}_{2} \mathrm{Cl}_{4}$ mixing ratios and $\left[\mathrm{C}_{2} \mathrm{Cl}_{4}\right] /\left[\mathrm{C}_{2} \mathrm{HCl}_{3}\right]$ individually 142 for TMRX-ET, which is located $\sim 1.7 \mathrm{~km}$ south of the DSW.

a. b.

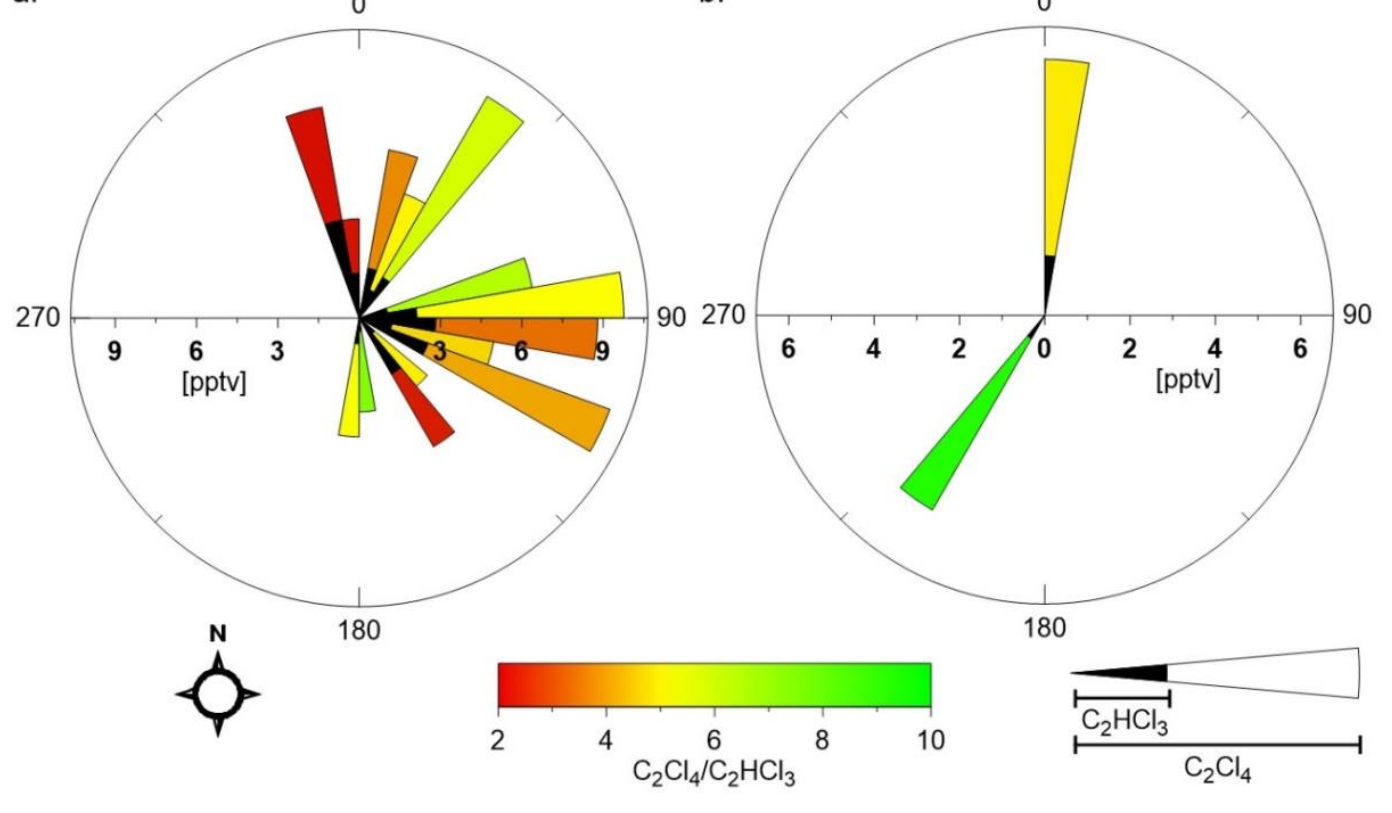

153 Figure S2. Anthropogenic impact on measured VHOCs. Presented are the $\mathrm{C}_{2} \mathrm{Cl}_{4}$ and $\mathrm{C}_{2} \mathrm{HCl}_{3}$ mixing ratios vs. $10^{\circ}$ 154 wind direction (WDD) sectors, as well as the $\left[\mathrm{C}_{2} \mathrm{Cl}_{4}\right] /\left[\mathrm{C}_{2} \mathrm{HCl}_{3}\right]$ which is indicated by color, for all sites to the north of the Dead Sea Works (a) and TMRX-ET, which is located to the south of the Dead Sea Works (b). 
Figure S2a shows elevated contribution from the eastern sector (apparently from the 157 eastern coast of the Dead Sea, i.e., from Jordan) to the mixing ratios of $\mathrm{C}_{2} \mathrm{Cl}_{4}$ and to some 158 extent also to those of $\mathrm{C}_{2} \mathrm{HCl}_{3}$, compared to the other wind sectors. It does not support 159 contribution from the DSW which is located some dozens of kilometers to the south of these 160 sites (see Fig. 1 in the main text). Potentially, the relatively small $\left[\mathrm{C}_{2} \mathrm{Cl}_{4}\right] /\left[\mathrm{C}_{2} \mathrm{HCl}_{3}\right]$ from the $161140^{\circ}-150^{\circ}$ sector, suggesting relatively fresh emission, could be attributed to emission from the 162 DSW, which could indicate no elevated contribution to $\mathrm{C}_{2} \mathrm{HCl}_{3}$ or $\mathrm{C}_{2} \mathrm{Cl}_{4}$ from the DSW. 163 Furthermore, Fig. S2b demonstrates that the single measurement that can be attributed to 164 emission from the DSW (from the $0^{\circ}-10^{\circ}$ sector) for TMRX-ET, which is significantly closer 165 to the DSW than the other sites, corresponds to significantly less fresh emission compared with 166 those measured at the other sites for the $140^{\circ}-150^{\circ}$ sector. Moreover, the mixing ratios of both $167 \mathrm{C}_{2} \mathrm{HCl}_{3}$ and $\mathrm{C}_{2} \mathrm{Cl}_{4}$, which correspond to the $0^{\circ}-10^{\circ}$ wind direction sector (Fig. S2b), seem to 168 not be elevated compared to the corresponding mixing ratios measured at the other sites. 169 Nevertheless, additional measurements are required to better analyze the contribution of 170 anthropogenic origin to the mixing ratios of these species at the Dead Sea. 
173 Table S6. Summary of measured meteorological data during the different samplings. Summary of VHOCs over

174 the Dead Sea. The table shows the date, time, site name (and abbreviation), as well as the average and standard

175 deviation, in parentheses, for the following parameters: wind speed (WDS), wind direction (WDD) and wind

176 vector (WDDV), temperature (T), relative humidity (RH), global solar radiation (GSR).

\begin{tabular}{|c|c|c|c|c|c|c|c|c|}
\hline $\begin{array}{c}\text { Date } \\
\mathrm{dd} / \mathrm{m} / \mathrm{yyyy}\end{array}$ & $\begin{array}{l}\text { Time } \\
\text { (local) }\end{array}$ & $\begin{array}{c}\text { Site name / } \\
\text { measurement } \\
\text { abbreviation }^{\text {a }}\end{array}$ & $\begin{array}{l}\text { WDS } \\
(\mathrm{m} / \mathrm{s})\end{array}$ & $\begin{array}{c}\text { WDD } \\
(\circ)\end{array}$ & $\begin{array}{c}\text { WDDV } \\
(\circ)\end{array}$ & $\begin{array}{c}\mathbf{T} \\
\left({ }^{\circ} \mathbf{C}\right)\end{array}$ & $\begin{array}{l}\mathrm{RH} \\
(\%)\end{array}$ & $\begin{array}{c}\text { GSR } \\
(\%)\end{array}$ \\
\hline $20 / 4 / 2016$ & $\begin{array}{c}08: 45- \\
08: 55\end{array}$ & $\begin{array}{l}\text { BARE-MSMR / } \\
\text { BARE-MSMR-1 }\end{array}$ & $\begin{array}{c}6.39 \\
(0.52)\end{array}$ & $\begin{array}{l}10.16 \\
(1.57)\end{array}$ & $\begin{array}{l}10.11 \\
(1.22)\end{array}$ & $\begin{array}{l}29.63 \\
(0.24)\end{array}$ & $\begin{array}{l}28.48 \\
(0.99)\end{array}$ & $\begin{array}{l}618.4 \\
(13.0)\end{array}$ \\
\hline $21 / 4 / 2016$ & $\begin{array}{l}08: 45- \\
08: 55\end{array}$ & $\begin{array}{c}\text { WM-KLY / } \\
\text { WM-KLY-1 }\end{array}$ & $\begin{array}{c}6.27 \\
(0.71) \\
\end{array}$ & $\begin{array}{l}13.65 \\
(3.19)\end{array}$ & $\begin{array}{l}13.91 \\
(5.52)\end{array}$ & $\begin{array}{l}27.72 \\
(0.12) \\
\end{array}$ & $\begin{array}{l}20.98 \\
(0.84) \\
\end{array}$ & $\begin{array}{l}332.5 \\
(12.1)\end{array}$ \\
\hline $02 / 5 / 2016$ & $\begin{array}{c}08: 45- \\
08: 55\end{array}$ & $\begin{array}{l}\text { TMRX-ET / } \\
\text { TMRX-ET-1 }\end{array}$ & $\begin{array}{c}1.87 \\
(0.29)\end{array}$ & $\begin{array}{l}214.99 \\
(16.78)\end{array}$ & $\begin{array}{l}214.40 \\
(12.35)\end{array}$ & $\begin{array}{l}26.50 \\
(0.12)\end{array}$ & $\begin{array}{l}48.76 \\
(0.46)\end{array}$ & $\begin{array}{l}538.1 \\
(8.9)\end{array}$ \\
\hline $03 / 5 / 2016$ & $\begin{array}{c}08: 45- \\
08: 55\end{array}$ & $\begin{array}{l}\text { WM-KLY / } \\
\text { WM-KLY-2 }\end{array}$ & $\begin{array}{c}3.74 \\
(0.44)\end{array}$ & $\begin{array}{c}340.19 \\
(4.30)\end{array}$ & $\begin{array}{l}340.33 \\
(10.95)\end{array}$ & $\begin{array}{l}25.93 \\
(0.12)\end{array}$ & $\begin{array}{l}45.71 \\
(0.46)\end{array}$ & $\begin{array}{c}225.5 \\
(8.9)\end{array}$ \\
\hline $25 / 5 / 2016$ & $\begin{array}{c}08: 30^{-} \\
08: 40 \\
\end{array}$ & $\begin{array}{l}\text { BARE-MSD / } \\
\text { BARE-MSD-1 }\end{array}$ & $\begin{array}{c}1.86 \\
(0.27) \\
\end{array}$ & $\begin{array}{l}104.00 \\
(15.65) \\
\end{array}$ & $\begin{array}{l}103.92 \\
(19.44) \\
\end{array}$ & $\begin{array}{l}26.46 \\
(0.13) \\
\end{array}$ & $\begin{array}{l}48.26 \\
(0.52) \\
\end{array}$ & $\begin{array}{l}518.9 \\
(10.6) \\
\end{array}$ \\
\hline $26 / 5 / 2016$ & $\begin{array}{c}08: 30^{-} \\
08: 40\end{array}$ & $\begin{array}{l}\text { BARE-MSD / } \\
\text { BARE-MSD-2 }\end{array}$ & $\begin{array}{c}2.56 \\
(0.65)\end{array}$ & $\begin{array}{l}141.03 \\
(12.26)\end{array}$ & $\begin{array}{l}141.71 \\
(19.42)\end{array}$ & $\begin{array}{l}28.48 \\
(0.09)\end{array}$ & $\begin{array}{l}34.06 \\
(0.49)\end{array}$ & $\begin{array}{l}497.6 \\
(10.1)\end{array}$ \\
\hline $30 / 5 / 2016$ & $\begin{array}{c}12: 00- \\
12: 10\end{array}$ & $\begin{array}{c}\text { WM-ET / } \\
\text { TMRX-ET-2 }\end{array}$ & $\begin{array}{c}2.75 \\
(0.52)\end{array}$ & $\begin{array}{c}9.42 \\
(18.42)\end{array}$ & $\begin{array}{c}9.40 \\
(13.50)\end{array}$ & $\begin{array}{l}31.16 \\
(0.32)\end{array}$ & $\begin{array}{l}29.94 \\
(0.89)\end{array}$ & $\begin{array}{c}1044.5 \\
(2.4)\end{array}$ \\
\hline $31 / 5 / 2016$ & $\begin{array}{c}12: 00^{-} \\
12: 10\end{array}$ & $\begin{array}{l}\text { BARE-MSMR / } \\
\text { BARE-MSMR-2 }\end{array}$ & $\begin{array}{c}2.90 \\
(0.34)\end{array}$ & $\begin{array}{l}78.26 \\
(8.52)\end{array}$ & $\begin{array}{l}78.47 \\
(5.40)\end{array}$ & $\begin{array}{l}30.83 \\
(0.29)\end{array}$ & $\begin{array}{l}30.64 \\
(0.97)\end{array}$ & $\begin{array}{c}1027.4 \\
(1.6)\end{array}$ \\
\hline $11 / 7 / 2016$ & $\begin{array}{c}12: 00- \\
12: 20\end{array}$ & $\begin{array}{l}\text { BARE-MSD / } \\
\text { BARE-MSD-3 }\end{array}$ & $\begin{array}{l}1.91 \\
(0.65)\end{array}$ & $\begin{array}{c}12.12 \\
(39.32)\end{array}$ & $\begin{array}{c}21.69 \\
(12.47)\end{array}$ & $\begin{array}{l}36.60 \\
(0.35)\end{array}$ & $\begin{array}{l}33.96 \\
(0.87)\end{array}$ & $\begin{array}{c}1000.7 \\
(6.4)\end{array}$ \\
\hline $11 / 7 / 2016$ & $\begin{array}{c}18: 00^{-} \\
18: 20\end{array}$ & $\begin{array}{l}\text { BARE-MSD / } \\
\text { BARE-MSD-4 }\end{array}$ & $\begin{array}{c}3.35 \\
(0.38) \\
\end{array}$ & $\begin{array}{l}350.53 \\
(9.46)\end{array}$ & $\begin{array}{l}350.61 \\
(9.53)\end{array}$ & $\begin{array}{l}40.19 \\
(0.15) \\
\end{array}$ & $\begin{array}{l}22.46 \\
(0.30) \\
\end{array}$ & $\begin{array}{c}260.00 \\
(24.9)\end{array}$ \\
\hline $21 / 2 / 2017$ & $\begin{array}{c}11: 20- \\
11: 40\end{array}$ & $\begin{array}{l}\text { COAST-TKM-SD / } \\
\text { COAST-TKM-SD-w }\end{array}$ & $\begin{array}{c}2.36 \\
(0.55)\end{array}$ & $\begin{array}{l}111.13 \\
(12.84)\end{array}$ & $\begin{array}{l}111.95 \\
(13.77)\end{array}$ & $\begin{array}{l}18.50 \\
(0.15)\end{array}$ & $\begin{array}{l}37.32 \\
(0.61)\end{array}$ & $\begin{array}{l}790.1 \\
(4.4)\end{array}$ \\
\hline $22 / 2 / 2017$ & $\begin{array}{l}11: 00- \\
11: 20\end{array}$ & $\begin{array}{l}\text { COAST-TKM-LD / } \\
\text { COAST-TKM-LD-w }\end{array}$ & $\begin{array}{l}2.91 \\
(0.52)\end{array}$ & $\begin{array}{l}29.85 \\
(12.11)\end{array}$ & $\begin{array}{l}30.66 \\
(5.35)\end{array}$ & $\begin{array}{l}20.29 \\
(0.16)\end{array}$ & $\begin{array}{l}34.01 \\
(0.28)\end{array}$ & $\begin{array}{l}454.1 \\
(47.9)\end{array}$ \\
\hline $28 / 2 / 2017$ & $\begin{array}{c}11: 20^{-} \\
11: 40\end{array}$ & $\begin{array}{l}\text { COAST-EGD-SD / } \\
\text { COAST-EGD-SD-w }\end{array}$ & $\begin{array}{l}7.90 \\
(0.64)\end{array}$ & $\begin{array}{l}13.60 \\
(3.19)\end{array}$ & $\begin{array}{l}13.61 \\
(1.96)\end{array}$ & $\begin{array}{l}24.71 \\
(0.13)\end{array}$ & $\begin{array}{l}27.70 \\
(1.92)\end{array}$ & $\begin{array}{l}314.1 \\
(82.1)\end{array}$ \\
\hline $01 / 3 / 2017$ & $\begin{array}{l}11: 07- \\
11: 27\end{array}$ & $\begin{array}{l}\text { COAST-EGD-MD / } \\
\text { COAST-EGD-MD-w }\end{array}$ & $\begin{array}{c}8.23 \\
(1.05) \\
\end{array}$ & $\begin{array}{l}28.06 \\
(5.08)\end{array}$ & $\begin{array}{l}28.09 \\
(1.34)\end{array}$ & $\begin{array}{l}22.12 \\
(0.38) \\
\end{array}$ & $\begin{array}{l}55.06 \\
(0.58) \\
\end{array}$ & $\begin{array}{l}506.9 \\
(93.9) \\
\end{array}$ \\
\hline $02 / 3 / 2017$ & $\begin{array}{l}11: 00- \\
11: 20\end{array}$ & $\begin{array}{l}\text { COAST-EGD-LD / } \\
\text { COAST-EGD-LD-w }\end{array}$ & $\begin{array}{c}8.32 \\
(0.96)\end{array}$ & $\begin{array}{l}182.17 \\
(5.96)\end{array}$ & $\begin{array}{l}182.28 \\
(6.38)\end{array}$ & $\begin{array}{l}23.49 \\
(0.19)\end{array}$ & $\begin{array}{l}44.43 \\
(0.45)\end{array}$ & $\begin{array}{l}345.5 \\
(68.0)\end{array}$ \\
\hline $25 / 4 / 2017$ & $\begin{array}{c}11: 30- \\
11: 50\end{array}$ & $\begin{array}{c}\text { COAS-EGD-SD / } \\
\text { COAST-EGD-SD-s }\end{array}$ & $\begin{array}{c}5.40 \\
(0.31)\end{array}$ & $\begin{array}{l}16.30 \\
(3.97)\end{array}$ & $\begin{array}{l}16.33 \\
(2.88)\end{array}$ & $\begin{array}{l}23.65 \\
(0.16)\end{array}$ & $\begin{array}{l}42.99 \\
(1.03)\end{array}$ & $\begin{array}{l}1031.5 \\
(0.95)\end{array}$ \\
\hline $26 / 4 / 2017$ & $\begin{array}{l}11: 00 \\
11: 20\end{array}$ & $\begin{array}{l}\text { COAST-EGD-MD / } \\
\text { COAST-EGD-MD-s }\end{array}$ & $\begin{array}{l}2.15 \\
(0.25)\end{array}$ & $\begin{array}{l}82.45 \\
(8.05)\end{array}$ & $\begin{array}{l}82.15 \\
(6.42)\end{array}$ & $\begin{array}{l}26.37 \\
(0.29)\end{array}$ & $\begin{array}{l}33.67 \\
(0.38)\end{array}$ & $\begin{array}{l}831.5 \\
(10.8)\end{array}$ \\
\hline $27 / 4 / 2017$ & $\begin{array}{l}11: 00- \\
11: 20\end{array}$ & $\begin{array}{l}\text { COAST-EGD-LD / } \\
\text { COAST-EGD-LD-s }\end{array}$ & $\begin{array}{l}2.39 \\
(0.77)\end{array}$ & $\begin{array}{l}104.12 \\
(23.19)\end{array}$ & $\begin{array}{c}98.01 \\
(14.98)\end{array}$ & $\begin{array}{l}27.03 \\
(0.19)\end{array}$ & $\begin{array}{l}42.27 \\
(0.54)\end{array}$ & $\begin{array}{c}707.9 \\
(153.8)\end{array}$ \\
\hline 03/5/2017 & $\begin{array}{c}12: 10- \\
12: 30\end{array}$ & $\begin{array}{l}\text { COAST-TKM-SD / } \\
\text { COAST-TKM-SD-s }\end{array}$ & $\begin{array}{c}2.80 \\
(0.41)\end{array}$ & $\begin{array}{l}174.49 \\
(19.14)\end{array}$ & $\begin{array}{l}174.76 \\
(21.93)\end{array}$ & $\begin{array}{l}28.44 \\
(0.33)\end{array}$ & $\begin{array}{l}36.21 \\
(1.05)\end{array}$ & $\begin{array}{c}961.6 \\
(7.4)\end{array}$ \\
\hline $04 / 5 / 2017$ & $\begin{array}{c}10: 30- \\
10: 50\end{array}$ & $\begin{array}{l}\text { COAST-TKM-LD / } \\
\text { COAST-TKM-LD-s }\end{array}$ & $\begin{array}{c}2.70 \\
(0.43)\end{array}$ & $\begin{array}{l}132.88 \\
(16.62)\end{array}$ & $\begin{array}{l}132.16 \\
(17.79)\end{array}$ & $\begin{array}{l}26.11 \\
(0.25)\end{array}$ & $\begin{array}{l}45.25 \\
(1.21)\end{array}$ & $\begin{array}{l}871.8 \\
(13.1)\end{array}$ \\
\hline
\end{tabular}




\section{References}

Carpenter, L. J., Reimann, S., Burkholder, J. B., Clerbaux, C., Hall, B. D., Hossaini, R., Laube, J. C., and Yvon-Lewis, S. A.: Ozone-depleting substances (ODSs) and other gases of interest to the Montreal Protocol, Chapter 1 in Scientific Assessment of Ozone Depletion: 2014, Global Ozone Research and Monitoring Project - Report No. 55, World Meteorological Organization, Geneva, Switzerland, 2014.

Olaguer, E. P.: The distribution of the chlorinated solvents dichloromethane, perchloroethylene, and trichloroethylene in the global atmosphere, Environ Sci Pollut R, 9, 175182,10.1065/espr2001.06.080, 2002.

Quack, B., and Suess, E.: Volatile halogenated hydrocarbons over the western Pacific between 43 degrees and 4 degrees N, J Geophys Res-Atmos, 104, 1663-1678, Doi 10.1029/98jd02730, 1999.

Schuepp, P. H., Leclerc, M. Y., Macpherson, J. I., and Desjardins, R. L.: Footprint Prediction of Scalar Fluxes from Analytical Solutions of the Diffusion Equation, Bound-Lay Meteorol, 50, 353-373, 1990.

Singh, H. B., Thakur, A. N., Chen, Y. E., and Kanakidou, M.: Tetrachloroethylene as an indicator of low $\mathrm{Cl}$ atom concentrations in the troposphere (vol 23, pg 1529, 1996), Geophys Res Lett, 23, 27132713, Doi 10.1029/96gl02556, 1996.

Zhou, Y., Varner, R. K., Russo, R. S., Wingenter, O. W., Haase, K. B., Talbot, R., and Sive, B. C.: Coastal water source of short-lived halocarbons in New England, J Geophys Res-Atmos, 110, Artn D2130210.1029/2004jd005603, 2005. 